\title{
Emotional Interaction Through Physical Movement
}

\author{
Jong-Hoon Lee ${ }^{1,2}$, Jin-Yung Park ${ }^{1}$, and Tek-Jin Nam ${ }^{1}$ \\ ${ }^{1}$ Collaboration and Interaction Design Research Lab. \\ Department of Industrial Design, Korea Advanced Institute of Science and Technology, \\ 373-1, Guseong-dong, Useong-gu, Daejeon, Korea \\ ${ }^{2}$ INNOIZ Inc., Seoul, Korea \\ \{rniro, vanilla0, tjnam\}@kaist.ac.kr
}

\begin{abstract}
As everyday products become more intelligent and interactive, there are growing interests on the methods to improve emotional value attached to the products. This paper presents a basic method of using temporal and dynamic design elements, in particular physical movements, to improve the emotional value of products. To utilize physical movements in design, a relation framework between movement and emotion was developed as the first step of the research. In the framework, the movement representing emotion was structurized in terms of three properties; velocity, smoothness and openness. Based on this framework, a new interactive device, 'Emotion Palpus', was developed, and a user study was also conducted. The result of the research is expected to improve emotional user experience when used as a design method or directly applied to design practice as an interactive element of products.
\end{abstract}

Keywords: Emotion, Physical Movement Design, Interaction Design, Interactive Product Design, Design Method.

\section{Introduction}

Advanced technology has reduced the limitations in making products. Companies pay more attention to the customer's diverse needs and lifestyles. The emotional value of the product, which can improve user experiences in HCI, is becoming more important for the success of products.

The conventional and representative design method to enhance the emotional value of the product is to advance its aesthetic form. This is usually done by applying design principles for the organization of ordinary design elements, such as form, material and color. So far, the design elements have been mainly visual and static.

However, as products are getting intelligent and more interactive by advanced technology, new design elements have emerged. One kind is the dynamic and temporal design elements. Especially physical movement is an interesting dynamic element for expressing functional or emotional status of products. For example, in everyday life, a fluttering leaf in a storm calls a distressing feeling, and a slowly moving pendulum weight looks courteous and generous. Physical movement can easily attract people's attention by visually changing its surroundings and can be independently expressed in the tangible space unlike graphical movement. 
Nevertheless, it is hard to represent its scale or shape due to the 3 dimensional form and material of the product, and difficult to implement it mechanically due to physical limitations. Detailed design methods of utilizing physical movement for emotional value of product, however, hasn't been thoroughly studied.

The objective of this study is to investigate the method of using dynamic design elements, particularly physical movement, to improve the emotional value and expression of a product. To do this, we first analyzed the related work of movement application by literature review. The relationship between emotion and movement in the existing studies was investigated, and in-depth characteristics of emotion and movement were studied to reify the relationship. Then, we conducted field research for the development of the relationship. From the result of the basic research, a relation framework between emotion and movement was developed. To apply the framework in product design and human computer interaction, we developed a new interactive device that can express the emotion of a product. Evaluating the framework using the device and developing application scenario were conducted.

\section{Related Work of Movement Application}

In the visual communication design field, various studies have conducted meaningful effects of integrating dynamic movement with static images. Vaughan(1997) proposed that understanding motion expression of performance art can be helpful in applying movement as a design element in GUI [1]. Uekita(2000) introduced the method of improving emotion by designing the movement of Kinetic Typography [2]. These studies, though, haven't presented the detail method to design movements for a certain emotion. In the product design field, some studies show application examples using movement as a design element. Weerdesteijn(2005) applied movements for the education assistant device that enables children to learn emotional expression through their body [3]. Physical movements have also been proposed applying to a communication device [4] or ambient display [5], [6]. Even though these works tried to utilize movement as a design element in various ways, few have studied the systematic methods or application of physical movement as a design element.

The way to apply movement to product design can be divided in two parts: one is to let the product itself move, and the other is to provide a separate moving part to the product. Even though few examples of applying movement to the product itself, such as Tanks Tail ${ }^{1}$ and $\mathrm{Nabaztag}^{2}$, have been commercialized recently, these stay on the level of using movement for people's concentration.

In the non-design fields, more works have been done to understand and represent the relationship between movement and emotion, but it is still difficult to find a proper example of applying movement to design from these studies [7], [8], [9]. Therefore, to design efficient physical movements, it is essential to study embodying the relationship between emotion and movement of various fields. Additionally, the study of utilizing this for practical development of products from the design perspective is necessary as well.

\footnotetext{
${ }^{1}$ http://www.k-wako.co.jp/enter/tail.html

${ }^{2}$ http://www.nabaztag.com/vl/FR/index.html
} 


\section{Theoretical Background of Relation Between Emotion and Movement}

\subsection{The Formal Components of Movement}

Movement consists of several formal components. Kepes and Moholy-Nagy, polytechnic engineers, classified them as following [10];

1) Rhythm: intervals of time between a motion and the next motion

2) Beat: rhythmical flow or rhythmic pace.

3) Sequence: relating to both rhythm and beat, explaining time based events

4) Direction: generated when an object has sequential movement in space

Aside from these four, path, volume, and speed are frequently referred as well.

\subsection{The Characteristic of Emotion}

In order to structurally understand the relationship between multifaced emotion and movement, it is important to understand the criteria in classifying emotion.

In the studies of emotion, sections of emotion are classified by Basic Categories and Multidimensional Categories. The former is grouping similar emotions within discrete categories, while the latter is distinguished by emotion space with various axis of more than 2-dimension ${ }^{3}$. Here, emotion space is generated by bi-polar dimensions, where each axis has two opposing adjectives as the emotion scale.

The most representative one of diverse multidimensional models is the Circumplex Model. The generalized model of the existing Circumplex Models by Russell is composed with Pleasantness axis and Activation axis [11]. These two emotion components were selected for the foundation of structuralizing the relationship between movement and emotion.

\subsection{The Emotion-Movement Relation Framework}

\section{The Literature Study Analysis for the Emotion-Movement Relation}

The speed of movement is related to 'Activation', so faster movement can present more activated emotion [9]. Boone and Cunningham(2001)[7] deduced the relationship between volume and sequence of movement. He mentioned that big and smooth movement was presented from pleasant or joyful emotion, and dreadful or sorrowful emotion caused shrinking movement. Negative emotion brought about jerky movements in uneven beats as well.

We applied these results to two emotion components of the Circumplex Model; 'Activation' is related to speed and volume of movement, and both ends of the 'Pleasantness' axis are related to smooth or jerky movement.

\section{The Field Study Analysis by In-Depth Interview}

A field study was conducted to interpret the connection between movement and emotion in the literature study. All participants were choreographers who create

\footnotetext{
${ }^{3}$ Kim, J., Moon, J., Designing towards emotional usability in customer interface-strustworthiness of cyber-banking system interfaces, Interacting with Computers, Vol. 10, pp. 1-29, 1998.
} 
movement for performance or dance directors who teach dancing. They had in-depth interview with questionnaires about emotional representation through movements in their field ${ }^{4}$. The result of the interview shows that emotions distinguished by 'Activation' and 'Pleasantness' in the Circumplex Model can be expressed as formal components of movement, such as speed, sequence, rhythm, and volume.

\section{The Emotion-Movement Relation Framework}

Based on the preliminary researches, an Emotion-Movement relation framework was developed. Settled rhythm and beat can be integrated as 'Velocity', and sequence and uneven rhythm can be interpreted as 'Smoothness'. Direction and volume are combined as 'Openness' including constriction and expansion.

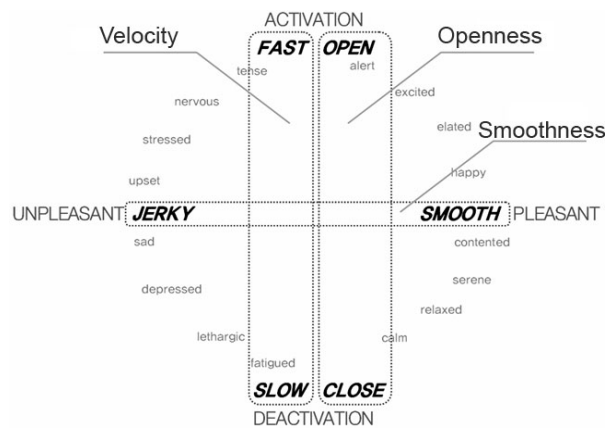

Fig. 1. The Emotion-Movement Relation Framework

Fig. 1 is the framework to design relevant physical movements for emotional expression, proposed in this study. After defining the relative emotion scales on each axis and combining proper degree of three movement components, the intended emotion is finally presented.

\section{Application Prototype: Emotion Palpus}

\subsection{Concept of Emotion Palpus}

In order to explore how this framework can be used in a new interactive product or in existing products by adding physical movement features, we developed a new interactive device called Emotion Palpus. It is a physical device that can generate physical movements to express various emotions. Its movement speed and movement type can be controlled to adjust 'Velocity' and 'Smoothness' of the framework, so it can be used as a prototyping and test platform. Its metaphor is from a palpus of living

\footnotetext{
${ }^{4}$ The interview had progressed for two hours per a day from the $18^{\text {th }}$ of June to the $20^{\text {th }}$ of June in 2006. Twenty interviewees were selected among the professional, who have more than 20 years work experience. One director of Korean dance co. and two ballet teachers participated separately.
} 
creatures (insects, snails and so on). It helps communicate through moving feelers, attached to existing products, such as PC monitors, telephones, and audio devices.

In addition to the hardware prototype, software interface was also developed to enable the user to make emotional movements by controlling velocity, smoothness, and openness of the physical object's movement. Fig. 2 shows its system structure.

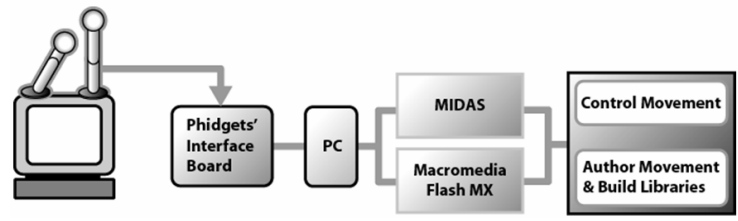

Fig. 2. The System structure of Emotion Palpus

\subsection{Components and Implement}

\section{1) Hardware}

The hardware part has a simple shape to minimize other aspects like form, which can influence emotion. It has a bar-shaped structure, consisting of three servo-motors, which were used for vertical piston movement with a spring, back-and-forth and right-and-left movement with two joints (Fig. 3). This hardware is used to express various movements while minimizing structural restrictions.

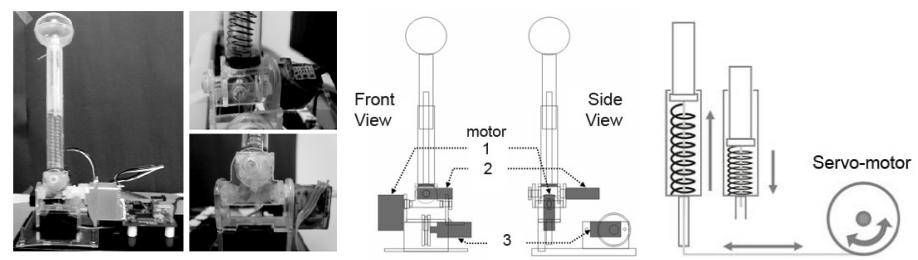

Fig. 3. The hardware prototype of Emotion Palpus and its structure drawing

The horizontal movement generated by the rotation of servo-motors is swapped to vertical movement, and piston movement is embodied by coiled spring. The rotating movements back-and-forth and right-and-left are presented by the rotation to the $\mathrm{X}$-axis and the rotation to the $\mathrm{Y}$-axis in the 3-dimensional space. To connect the hardware with the PC, two '4-Motor PhidgetServo' interfaces by Phidgets ${ }^{5}$ were used.

\section{2) Software}

The software part of Emotion Palpus is to control the movement of hardware through GUI on the screen. The movement made by a user can be saved and reused. A user can easily present speed, openness and smoothness by controlling the angle of the servo-motors on the time-line graph in its user interface. To control three servomotors of a device, each motor can be controlled with a modular controller (Fig. 4).

\footnotetext{
${ }^{5}$ Phidgets Inc., PhidgetsTM, http://www.phidgets.com
} 


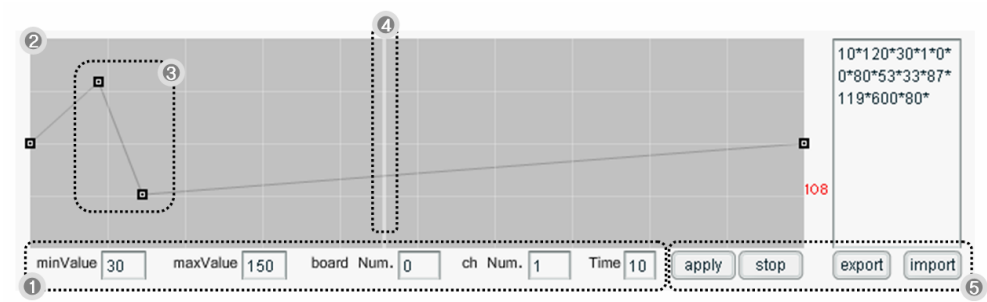

Fig. 4. One modular graphical user interface of Emotion Palpus's software

Input area: the maximum degree of the motor's angle, the minimum degree, the interface board number, channel number, running time.

Work place: the area to control the angle graph on the time-line The graph editor: the graph representing angle degree at the point Time bar

Action buttons: play, pause, output of text code, input of text code

Flash 8.0 and MIDAS, interactive prototyping tools, were used for the embodiment of the software module and the motor-control. MIDAS supports components that can help install complex hardware with simple scripts and control interface boards with Flash.

\section{Evaluation of the Framework with Emotion Palpus}

We conducted two experiments with Emotion Palpus to verify the validity of the Emotion-Movement Relation Framework.

\subsection{Experiment 1}

\section{Experiment Design}

The purpose of the first experiment was to know the relation between two elements of emotion and three elements of movement. The emotional effect from the movement elements -velocity, openness and smoothness- was examined and analyzed.

- Velocity: 5 levels to differentiate each time of a circular movement basis

- Openness: 5 levels to differentiate width and height of a circular movement basis

- Smoothness: 5 levels of circular movement without acceleration to movement with four accelerations

15 movements with Emotion Palpus were used, representing different levels of each element (Fig. 5). For each level, 15 movie clips (740x480) were randomly presented with Windows Media Player on a 19 inch monitor display. Participants consisted of university students from 21 to 26 years old, 6 males and 4 females. All of them are novices at movement design. 


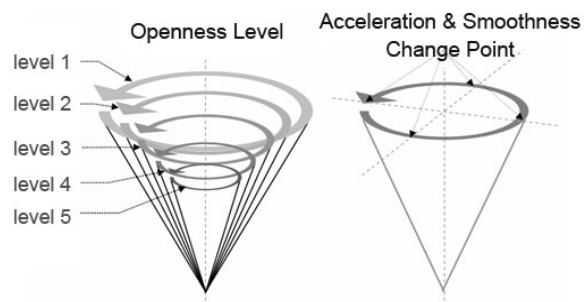

Fig. 5. The levels of 'Openness' and the change point of 'Acceleration and Smoothness'

\section{Procedure}

5 levels per each movement of all were provided and repeatedly presented three times. After watching one movie representing a level, participants selected the most appropriate emotion from the list in the questionnaire.

\section{Result}

The experiment was evaluated the relationship between emotion and movement element: velocity, openness and smoothness. The relationship of emotion and velocity or smoothness of movement was statistically significant with $(\mathrm{F}=99.538, \mathrm{p}=.000)$ and $(\mathrm{F}=14.570, \mathrm{p}=.000)$ from the each result of one-way ANOVA test. Because both velocity and emotion categories were ranking scales, we conducted the correlation analysis through the Spearman correlation coefficient. As a result, velocity has correlation with the 'Activation' axis of emotion on the positive direction (=.930, $>.01)$. For smoothness, positive correlation with 'Pleasantness' was shown $(=.760$, $>.01)$ However, no significant effect was found for openness $(\mathrm{F}=.587, \mathrm{p}>0.05)$.

\subsection{Experiment 2}

\section{Experiment Design}

The purpose of the second experiment was to validate that the emotion from the framework can be properly recognized by the movement elements on the framework. The 16 stimuli were selected with Emotion Palpus: eight for levels of velocitysmoothness combination and eight for levels of openness-smoothness combination. Fig. 6 shows eight areas of emotions intended from them.

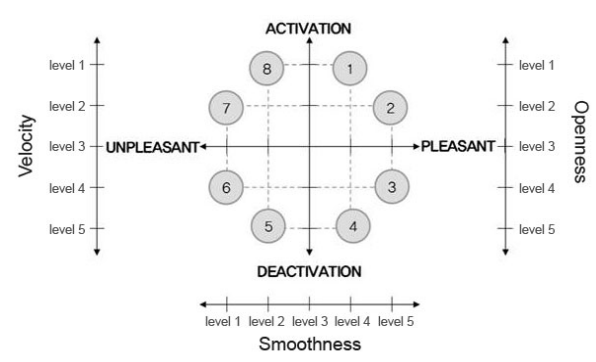

Fig. 6. Eight areas of the intended emotions in the Emotion-Movement Relation Framework 
16 Movie clips (740x460) were made for the experimental stimuli. 16 emotion adjectives on the Circumplex Model were chosen to evaluate each movement. The test was conducted with the same material and in the same environment with the first, except for the movie clips. Participants consisted of different people but in the same age or gender range with the participants of the first test.

\section{Procedure}

Each of the 16 stimuli was iteratively presented three times. Participants wrote the answer in the questionnaire sheet right after watching each movie clip.

\section{Result}

The results from a Velocity-Smoothness and Openness-Smoothness are shown in Fig. 7. In the left graph of Fig. 8, the selection rate of the intended emotion for high or low level of velocity unit is relatively high, whereas the selection rate of the intended emotion for middle level is low. This shows that velocity has significant influence on emotion. In the right graph, the answers are relatively spread out by and large.
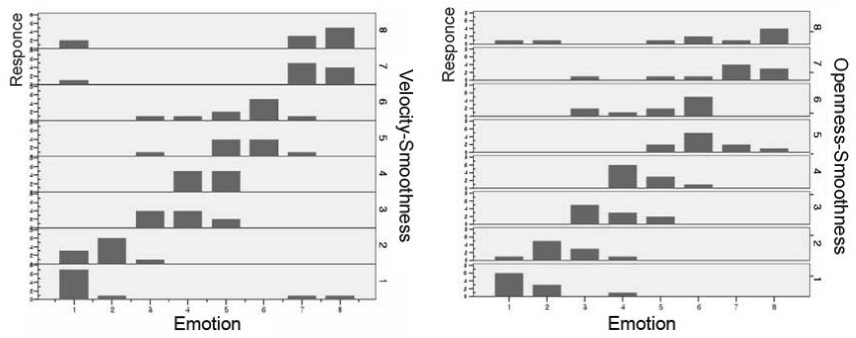

Fig. 7. The selection distribution graph of the intended emotions for 'Velocity-Smoothness' and 'Openness-Smoothness'

\subsection{Discussion}

As velocity and smoothness each show effective correlation in the first experiment with the 'Activation' axis and the 'Pleasantness' axis, the Emotion-Movement relation framework is validated. However, openness didn't show effective correlation with the framework from the result of the first experiment. The result of the second experiment presents that both Velocity-Smoothness unit and Openness-Smoothness unit do not correspond with the exact intended emotion but can influence round about the emotion. This is assumed to be because certain emotions were simply tested without any context. Specific context can help bring about certain emotions. Even if the Openness-Smoothness unit is less distinct than Velocity-Smoothness unit, it can lead to some of the intended emotions as well. This means openness affects the 'Activation' axis. From this result, we estimate that openness can be more effective at the moment of changing movement, from big movement to small movement or from small to big.

The result of the two experiments certifies the possibility of presenting relevant emotions in the intended area, compounding movement elements. This suggests that Emotion Palpus can be effectively utilized when designing expected emotions based on the framework. 


\section{Application Scenario of Emotion Palpus}

Emotion Palpus can be utilized alone or with various existing products, providing new emotional experiences. The following examples show its application scenarios.

In the trend of digital convergence and mobilization, Emotion Palpus can be attached into the cradle of a mobile device for emotionally an enhanced mobile experience (the picture (1) of Fig. 8). For example, a cradle with a mobile phone can emotionally provide information or messages by automatically analyzing alarm bells, phone bells or text messages. For the convergence mobile phone with DMB or MP3, the cradle can present diverse emotional movements by the display or sound of the device.

With the advance of network environment, on-line human to human communication is growing. A number of on-line firms have developed not only various chatting sites and messenger services but provided graphical emoticons or flash animations for more dynamic communication. Emotion Palpus can help tangible emotional interaction in this on-line environment by being implemented in some devices that provide on-line chatting service, such as PC, UMPC or mobile phone. The picture (2) of Fig. 8 shows a user chatting with physical emoticon connected to a laptop.

Portable Emotion Palpus can be also embedded into other variable devices -home audio system, TV, video phone or car dash-board etc. For instance, Emotion Palpus with dash-board can alert with sound and movement according to a driver's context by sensing the user's situation. Also, it can be installed in home electronics and provide emotional value. Pictures (3) and (4) of Fig. 8 show its concept model.

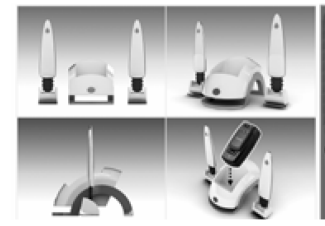

(1) Cradle Emotion Palpus

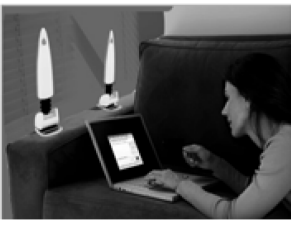

(2) Physical Emoticon

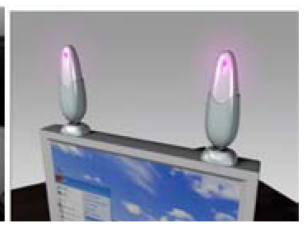

(3) Combination with PC (4) Combination with Audio

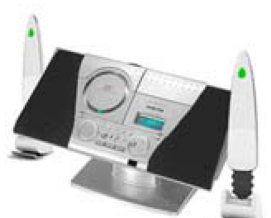

Fig. 8. The concept models of Emotion Palpus applications

\section{Conclusion and Future Work}

In this study, we introduced the framework of a new design method to apply physical movement as the element of improving emotional and functional value for products. In addition, we develop Emotion Palpus as its application prototype, which can emotionally present information or status of a product. This shows new potentials of applying physical movement to the novel media to express emotion. Emotion Palpus can enhance user emotional experience, installed in various products.

The result of this study offers the foundation of applying physical movement as a design element. For instance, it can be used for the emotional feedback in humanrobot interaction in the robot design field. Moreover, physical movement can perform 
as the interactive media in the user interface of diverse interactive products, which is currently represented just by buttons and displays. This implies that it can also provide the basis of designing promise products, which can maximize user emotional experience. This can contribute to the industry by bringing it in human life.

For future work, to efficiently use the framework and Emotion Palpus for practical design process and rich emotional expression, it is necessary to improve the structural aspects of Emotion Palpus. Implementing smaller size and volume of the device is necessary for various sized application devices as well. Additionally, a database library from the example implementation to diverse actual products will be useful for the design process of new interactive products.

Acknowledgement. This work was carried out when Jonghun Lee was a member of CIDR at KAIST. The work was supported by the Korea Research Foundation Grant funded by the Korean Government(MOEHRD). (KRF-2006-321-G00080).

\section{References}

1. Vaughan, L.C.: Understanding movement. In: Proceedings of CHI 1997, pp. 548-549 (1997)

2. Uekita, Y., Sakamoto, J., Furukata, M.: Composing motion grammar of kinetic typography. In: Proceedings of VL'00, pp. 91-92 (1997) (2000)

3. Weerdesteijn, W., Desemet, P., Gielen, M.: Moving Design: to design emotion through movement. The Design Journal 8, 28-40 (2005)

4. Moen, J.: Towards people based movement interaction and kinaesthetic interaction experiences. In: Proceedings of the 4th decennial conference on Critical computing: between sense and sensibility, pp. 121-124 (2005)

5. Ishii, H., Ren, S., Frei, P.: Pinwheels: Visualizing Information Flow in an Architectural Space. In: CHI '01(2001)

6. Jafarinaimi, N., Forlizzi, J., Hurst, A., Zimmerman, J.: Breakaway: An Ambient Display Designed to Change human Behavior. In: CHI '05 (2005)

7. Boone, R., Cunningham, J.: Chidren's Exprssion of Emotional Meaning in Music through expressive body movement. Journal of Nonverbal Behavior 25, 21-41 (2001)

8. Camurri, A., Poli, G., Leman, M., Volpe, G.: The MEGA Project: Analysis and Synthesis of Multisensory Expressive Gesture in Performing Art Applications. Journal of New Music Research 34, 5-21 (2005)

9. Pollick, F.E., Paterswon, H.M., Bruderlin, A., Sanford, A.J.: Perceiving affect from arm movement. Journal of Cognition 82, 51-61 (2001)

10. Bacigalupi, M.: The craft of movement in interaction design. In: Proceedings of the working conference on Advanced visual interfaces, L'Aquila, Italy (1998)

11. Russell, J.A.: A circumplex model of affect. Journal of Personality and Social Psychology 39, 1161-1178 (1980) 